\title{
DISTRIBUTION OF SURPLUS IN LIFE INSURANCE
}

\author{
By Henrik Ramlau-Hansen \\ Baltica Insurance Company Ltd., Ballerup, Denmark
}

\begin{abstract}
This paper discusses distribution of surplus in life insurance within a general Markov chain framework. A conservative interest rate and a conservative set of transition intensities are used for reserving purposes whereas more realistic assumptions are used for the purpose of distributing surplus. The paper examines various actuarial aspects of distributing surplus through either cash bonuses, terminal bonuses or increased benefits. The results are illustrated by some examples.
\end{abstract}

\section{KEYWORDS}

Distribution of surplus; bonus; with profits annuity policy; with profits disability policy.

\section{INTRODUCTION}

The traditional life policy is a participating policy with margins of safety built into the valuation elements to allow for protection for adverse deviations. Surplus or profit can, therefore, in most cases be expected to emerge over the life of a portfolio of business. A large proportion of the surplus is usually distributed to the policyholders as bonuses or dividends. This distribution of surplus may be carried out in various ways. One method provides cash payments or reduction of premiums as the surplus arises, or the accumulated value of the cash bonuses may be paid when the policy becomes a claim or expires. By this method, a separate savings account is attached to the policy and the surplus is credited to the account as it emerges. Another way of distributing surplus is through terminal bonuses paid only when the policy expires. By this method, only survivors get a share of the accumulated surplus. The third method, and perhaps the most widely used, is one in which the profit is distributed to the policyholders by means of increasing the insurance benefits. This method provides a gradual increase in the benefits granted under the policy.

It is believed that these three different ways of distributing surplus cover many of the methods used in practice. We shall in this paper discuss various actuarial aspects of the mentioned distribution methods. The idea is that the 
surplus should be distributed to those policyholders who contributed to the profit. Moreover, the distribution should be equitable, and the actuarial present value of the surplus generated by a policy should equal the actuarial present value of the bonuses paid to that same policy.

The results are discussed within a general Markov chain framework where an insurance policy is modelled as a time-inhomogeneous Markov chain, see e.g. Hoem $(1969,1988)$. The paper is motivated by BERGER (1939), SverDRUP (1969) and SIMONSEN (1970), who discussed some aspects of accumulation and distribution of surplus. Moreover, RAMLAU-HANSEN (1988) analysed the emergence of surplus using a general Markov chain and counting process framework.

\section{THE MARKov CHAIN MODEL}

We shall in the following consider life insurance policies which can be modelled by time-inhomogeneous Markov chains with finite state spaces. Hence, let $S(\cdot)$ denote the right-continuous sample path function of a time-inhomogeneous Markov chain with finite state space $I$, and assume that the process starts in a state $1 \in I$ at time 0 . The transition probabilities are denoted by $P_{i j}^{0}(s, t)=P(S(t)=j \mid S(s)=i), i, j \in I, s \leq t$, and the forces of transition $\mu_{i j}^{0}($.$) are defined by$

$$
\mu_{i j}^{0}(t)=\lim _{h \rightarrow 0^{+}} P_{i j}^{0}(t, t+h) / h, \quad i, j \in I, \quad i \neq j .
$$

The intensities are assumed to be integrable on compact intervals.

Consider an $n$-year insurance policy characterized by the following conditions :

1. While the policy stays in state $i$, premiums are paid continuously to the company at the rate $\pi_{i}(\cdot)$, i.e. $\pi_{i}(t) d t$ is paid during $[t, t+d t)$. Annuity benefits received by the insured while in state $i$ are denoted by $b_{i}(\cdot)$.

2. If the policy moves from state $i$ to state $j$ at time $t$, a lump sum benefit $B_{i j}(t)$ is paid to the insured immediately after time $t$.

3. When the policy expires at time $n$, the insured receives an amount $B_{i}(n)$ if the policy is in state $i$ at the maturity date.

The quantities $\pi_{i}(t), b_{i}(t), B_{i j}(t)$, and $B_{i}(n)$ are all assumed to be nonrandom. It should also be noted that we have restricted ourselves to continuous payment of premiums and annuities, benefits tied to transitions between different states and to maturity benefits. However, single premiums and other types of non-random payments can be incorporated easily. Note also that we have introduced different notation for premiums paid and annuity benefits received because the two types of payments are affected differently by surplus distribution. Moreover, we shall refer to the "standard" benefits $\left(b_{i}(t), B_{i j}(t)\right.$, $\left.B_{i}(n), i, j \in I, i \neq j\right)$ as one unit of benefits, because one of the distribution methods operates by increasing all benefits proportionally. Finally, expenses are not included explicitly but can be regarded as separate benefits. 
It is assumed that the company is making its valuations on the basis of a constant force of interest $\delta$ and a set of transition intensities $\mu_{i j}(\cdot)$. The basis $(\delta$, $\left.\mu_{i j}, i, j \in I, i \neq j\right)$ is often called the valuation basis of the first order, and we shall assume that the company is required to use this set of (conservative) assumptions in determining reserves and premiums. However, we shall assume that the actual force of interest is $\delta^{0}\left(\delta^{0}>\delta\right)$ and that the actual behaviour of the Markov chain is governed by the intensities $\mu_{i j}^{0}(\cdot)$. The elements $\left(\delta^{0}, \mu_{i j}^{0}\right.$, $i, j \in I, i \neq j$ ) are often called the second order basis, and we shall assume that surplus is distributed according to this set of (realistic) assumptions.

Given that the policy is in state $i$ at time $t$, let $V_{i}(t)$ denote the prospective premium reserve corresponding to the valuation basis of the first order. Moreover, let $S P_{i}(t)$ be the single premium or the actuarial present value of one unit of future benefits, provided that the policy is in state $i$ at time $t$. We shall also assume that the equivalence principle is followed, i.e. $V_{1}(0)=0$. The reserve $V_{i}(t)$ is given by

$$
\begin{aligned}
V_{i}(t)= & \sum_{j} \sum_{k \neq j} \int_{t}^{n} v^{u-t} P_{i j}(t, u) \mu_{j k}(u) B_{j k}(u) d u \\
& +\sum_{j} \int_{t}^{n} v^{u-t} P_{i j}(t, u)\left[b_{j}(u)-\pi_{j}(u)\right] d u \\
& +\sum_{j} v^{n-t} P_{i j}(t, n) B_{j}(n)
\end{aligned}
$$

where the $P_{i j}(s, t)$ 's are the transition probabilities corresponding to the intensities $\mu_{i j}(\cdot)$. A similar expression holds for $S P_{i}(t)$; just substitute 0 for $\pi_{j}(u)$ in (2.1). It is well known, see e.g. HoEm (1969), that $V_{i}(t)$ satisfies Thiele's differential equation

$$
\frac{d}{d t} V_{i}(t)=\delta V_{i}(t)+\pi_{i}(t)-b_{i}(t)-\sum_{j \neq i} \mu_{i j}(t) R_{i j}(t),
$$

where $R_{i j}(t)=V_{j}(t)+B_{i j}(t)-V_{i}(t)$ denotes the amount at risk associated with a transition from state $i$ to state $j$ at time $t$. Similarly, $S P_{i}(t)$ satisfies

$$
\frac{d}{d t} S P_{i}(t)=\delta S P_{i}(t)-b_{i}(t)-\sum_{j \neq i} \mu_{i j}(t)\left[S P_{j}(t)+B_{i j}(t)-S P_{i}(t)\right]
$$

\section{ACCUMULATION OF SURPLUS}

Assume in this section that no bonuses are paid and that the company just pays the promised benefits $b_{i}(t), B_{i j}(t)$, and $B_{i}(n)$ in return for the premiums $\pi_{i}(t)$. The average surplus or profit realized over the term of the policy may then be derived in the following way. Assume that the policy is in state $i$ at time $t$ and that the amount $V_{i}(t)$ has been reserved. Then during $[t, t+d t)$ the actual interest earned is $\delta^{0} d t V_{i}(t)$, the premiums and the annuity benefits are $\pi_{i}(t) d t$ 
and $b_{i}(t) d t$, respectively, and the expected net loss due to transitions out of state $i$ is $\sum_{j \neq i} \mu_{i j}^{0}(t) d t R_{i j}(t)$. However, the reserve needed at time $t+d t$, assuming the policy is still in state $i$, is $V_{i}(t+d t)$, and hence the net profit becomes

$\gamma_{i}(t) d t=\left(1+\delta^{0} d t\right) V_{i}(t)+\pi_{i}(t) d t-b_{i}(t) d t-\sum_{j \neq i} \mu_{i j}^{0}(t) d t R_{i j}(t)-V_{i}(t+d t)$.

This leads to

$$
\gamma_{i}(t)=\delta^{0} V_{i}(t)+\pi_{i}(t)-b_{i}(t)-\sum_{j \neq i} \mu_{i j}^{0}(t) R_{i j}(t)-\frac{d}{d t} V_{i}(t)
$$

and using (2.2) we get

$$
\begin{aligned}
\gamma_{i}(t) & =\left(\delta^{0}-\delta\right) V_{i}(t)+\sum_{j \neq i}\left(\mu_{i j}(t)-\mu_{i j}^{0}(t)\right) R_{i j}(t) \\
& =\Delta \delta V_{i}(t)+\sum_{j \neq i} \Delta \mu_{i j}(t) R_{i j}(t),
\end{aligned}
$$

introducing $\Delta \delta=\delta^{0}-\delta$ and $\Delta \mu_{i j}(t)=\mu_{i j}(t)-\mu_{i j}^{0}(t)$. Thus, assuming that the policy is in state $i$ at time $t$, surplus accumulates at the rate $\gamma_{i}(t)$, which, according to (3.1), is the sum of the excess interest earnings and the profit or loss associated with transitions out of state $i$. The actuarial present value at time 0 of the total surplus accumulated over $[0, t]$ during stays in the state $i$ is given by

$$
\Gamma_{i}(t)=\int_{0}^{t} e^{-\delta^{0} s} P_{1 i}^{0}(0, s) \gamma_{i}(s) d s,
$$

and the present value of the total surplus accumulated over $[0, t]$ is

$$
\Gamma(t)=\sum_{i} \Gamma_{i}(t)
$$

It should also be noted that

$$
\begin{aligned}
\Gamma(t)= & \sum_{i} \int_{0}^{t} e^{-\delta^{0} s} P_{1 i}^{0}(0, s)\left[\pi_{i}(s)-b_{i}(s)\right] d s \\
& -\sum_{i} \sum_{j \neq i} \int_{0}^{t} e^{-\delta^{0} s} P_{1 i}^{0}(0, s) \mu_{i j}^{0}(s) B_{i j}(s) d s \\
& -\sum_{i} e^{-\delta^{0} t} P_{1 i}^{0}(0, t) V_{i}(t),
\end{aligned}
$$


and

$$
\begin{aligned}
\Gamma_{i}(t)= & \sum_{k \neq i} \int_{0}^{t} e^{-\delta^{0} s} P_{1 k}^{0}(0, s) \mu_{k i}^{0}(s) V_{i}(s) d s \\
& +\int_{0}^{t} e^{-\delta^{0} s} P_{1 i}^{0}(0, s)\left[\pi_{i}(s)-b_{i}(s)\right] d s \\
& -\sum_{j \neq i} \int_{0}^{t} e^{-\delta^{0} s} P_{1 i}^{0}(0, s) \mu_{i j}^{0}(s)\left[B_{i j}(s)+V_{j}(s)\right] \\
& -e^{-\delta^{0} t} P_{1 i}^{0}(0, t) V_{i}(t),
\end{aligned}
$$

see e.g. Ramlau-Hansen (1988) formulas (4.1) and (4.10). Hence, $\Gamma(t)$ may be interpreted as the actuarial present value of the difference between the premiums received and the benefits and reserves that have to be provided. The gain $\Gamma_{i}(t)$ may be interpreted similarly.

For a broader discussion of surplus accumulation and in particular various stochastic aspects, see RAMLAU-HANSEN (1988). However, note that in RAMLAU-HANSEN (1988) $\Gamma(t)$ and $\Gamma_{i}(t)$ are random variables and not actuarial values.

\section{DISTRIBUTION OF SURPLUS}

\subsection{Cash bonuses}

It was shown in the previous section that the surplus accumulates at the rate $\gamma_{i}(t)$ in state $i$ at time $t$. Hence, the surplus may be distributed by simply paying the policyholder an annuity $\gamma_{i}(t)$ while the policy is in state $i$. These dividend payments may then supplement annuity benefits or partly offset premiums paid under the terms of the policy. The present value at time 0 of the total bonuses paid during $[0, t]$ is

$$
C(t)=\sum_{i} \int_{0}^{t} e^{-\delta^{0} s} Y_{i}(s) \gamma_{i}(s) d s,
$$

where $Y_{i}(s)=1$ if $S(s)=i$ and 0 otherwise. Note that the amount $C(t)$ is random, but $E C(t)=\Gamma(t)$. In practice, companies that pay cash bonuses do not pay the continuous annuities $\gamma_{i}(t)$, but they may distribute the surplus through annual instalments or by other means, cf. Section 5.1 .

The amount $C(t)$ may also be interpreted as the present value of the amount in a savings account attached to the insurance policy. During stays in state $i$, the account is then credited continuously at the rate $\gamma_{i}(t)$. Some companies do follow this procedure by deferring the payment of the cash bonus until the policy becomes a claim or expires. If the policy becomes a claim or expires at, say time $t$, then the amount $\exp \left(\delta^{0} t\right) C(t)$ is paid in addition to the policy 
benefits. If two or more lump sum payments are possible under the policy, the surplus may be distributed through a series of payments.

It should be noted that the distribution of surplus through periodic payments allows all policyholders to share in the profit.

\subsection{Terminal bonuses}

In this subsection we discuss a distribution method according to which the surplus is distributed to the policyholders only when the policies expire. No additional benefits are paid during the term of the policy, except at the maturity date. Hence, terminal bonuses may be used to enhance the maturity value of the policy.

It was shown in Section 3 that the actuarial present value of the total surplus accumulated during stays in a state $i$ is $\Gamma_{i}(n)$ given by (3.2). Hence, if this profit is to be distributed as a payment to those policyholders who are in state $i$ at time $n$, each should receive

$$
T_{i}(n)=\Gamma_{i}(n) /\left[e^{-\delta^{0} n} P_{1 i}^{0}(0, n)\right] .
$$

One might also limit the payment of bonuses to those survivors who are in the initial state at time $n$. Depending on the design of the policy, this practice may favour those policyholders who have not made any claims under the policy. In this situation, each of the survivors in state 1 should receive

$$
T(n)=\Gamma(n) /\left[e^{-\delta^{0} n} P_{11}^{0}(0, n)\right] .
$$

at time $n$.

However, it should be noted that by applying terminal bonuses only survivors are rewarded, and those who have died do not get a share of the profit, although they may actually have contributed to it. Hence, the method resembles in a way a tontine scheme, and this may explain why terminal bonuses are only used in connection with policies with a strong savings element.

\subsection{Increased benefits}

In this section we assume that the surplus is used to increase the policy benefits. This is one of the most common ways of distributing surplus in practice. We shall assume that all benefits are increased proportionally so that the original relationship between the benefits is preserved. Hence, the surplus is used as a single premium to purchase additional units of benefits, cf. Section 2 .

At issue, the net premium reserve is $V_{1}(0)=0$ and the policy provides the benefits $b_{i}(s), B_{i j}(s)$, for $s \geq 0$, and $B_{i}(n)$. Let us now assume that the policy is in state $i$ at time $t$ and that the policy entered this state at some time $t_{i}$. Moreover, assume that past surplus has been used to buy $D(t)$ units of additional benefits so that they are now promised to be $b_{j}^{*}(s)=b_{j}(s)(1+D(t)), B_{j k}^{*}(s)=$ $B_{j k}(s)(1+D(t))$, for $s \geq t$, and $B_{j}^{*}(n)=B_{j}(n)(1+D(t))$. The rate of increase 
of benefits at time $u$ is denoted by $d(u)$, i.e. $D(t)=\int_{0}^{t} d(u) d u$. It should be noted that $D(\cdot)$ is actually a stochastic process since it is a function of the sample path of the Markov chain. At time $0, D(t)$ is unknown because the future course of the policy is unknown.

Taking the increased benefits into account, the policy reserve is now

$$
V_{i}^{*}(t)=V_{i}(t)+D(t) S P_{i}(t),
$$

where both $V_{i}(t)$ and $S P_{i}(t)$ are calculated using the first order valuation basis, cf. (2.2)-(2.3). Hence, using arguments similar to the ones in Section 3, the average surplus that emerges at time $t$ is given by the rate

$$
\begin{aligned}
\gamma_{i}^{*}(t)= & \Delta \delta V_{i}^{*}(t)+\sum_{j \neq i} \Delta \mu_{i j}(t)\left[V_{j}^{*}(t)+B_{i j}^{*}(t)-V_{i}^{*}(t)\right] \\
= & \Delta \delta V_{i}(t)+\sum_{j \neq i} \Delta \mu_{i j}(t)\left[V_{j}(t)+B_{i j}(t)-V_{i}(t)\right] \\
& +D(t)\left\{\Delta \delta S P_{i}(t)+\sum_{j \neq i} \Delta \mu_{i j}(t)\left[S P_{j}(t)+B_{i j}(t)-S P_{i}(t)\right]\right\}
\end{aligned}
$$

using (4.4). Thus,

$$
\gamma_{i}^{*}(t)=\gamma_{i}(t)+D(t) \kappa_{i}(t)
$$

if we introduce $\kappa_{i}(t)=\Delta \delta S P_{i}(t)+\sum_{j \neq i} \Delta \mu_{i j}(t)\left[S P_{j}(t)+B_{i j}(t)-S P_{i}(t)\right]$.

The surplus $\gamma_{i}^{*}(t)$ is used to buy $d(t)$ units of additional benefits at a cost of $S P_{i}(t)$ per unit. Thus, we must have that

$$
d(t) S P_{i}(t)=\gamma_{i}(t)+D(t) \kappa_{i}(t),
$$

or

$$
D^{\prime}(t)=d(t)=q_{i}(t)+D(t) r_{i}(t),
$$

where $q_{i}(t)=\gamma_{i}(t) / S P_{i}(t)$ and $r_{i}(t)=\kappa_{i}(t) / S P_{i}(t)$. Equation (4.6) is a linear differential equation with solution

$$
\begin{aligned}
D(t)= & \int_{t_{i}}^{t} q_{i}(s) \exp \left(\int_{s}^{t} r_{i}(u) d u\right) d s \\
& +D\left(t_{i}\right) \exp \left(\int_{t_{i}}^{t} r_{i}(s) d s\right), \quad t \geq t_{i},
\end{aligned}
$$

which yields, in a closed form, an expression for the total increase of the benefits due to the emerging surplus. 
It should be noted that (4.7) holds only during the stay in state $i$. If the policy at some later time $t_{j}$ moves to state $j$ then a similar formula holds with $t_{j}$ and $j$ substituted for $t_{i}$ and $i$, respectively. Thus, the rate of increase of benefits depends on the current state of the policy, but the policyholder should not expect any sudden changes in the benefits because $D(\cdot)$ is a continuous function.

It should also be noted that in this section additional benefits are granted as the surplus is earned. In order to make this a prudent distribution method, it requires that at any time the future safety margins are sufficient to safeguard the company against any adverse experience. Moreover, since companies normally cannot reduce bonuses once they have been declared, it also requires surplus always to be positive, i.e. $\gamma_{i}^{*}(t)$ has to be positive. If this is not the case, distribution of surplus will have to be deferred, and the method above will have to be modified.

If the original policy is a single premium policy, then $V_{i}(t)=S P_{i}(t)$, $\kappa_{i}(t)=\gamma_{i}(t)$, and $q_{i}(t)=r_{i}(t)$. In this case, it follows from (4.7) that

$$
1+D(t)=\left(1+D\left(t_{i}\right)\right) \exp \left(\int_{t_{i}}^{t} r_{i}(u) d u\right), \quad t \geq t_{i}
$$

Finally, we shall see that $V_{i}^{*}(t)$ satisfies a second order differential equation although it was defined as a first order premium reserve, cf. (4.4). The reason is that the benefits are adjusted continuously. According to (4.4),

$$
\frac{d}{d t} V_{i}^{*}(t)=\frac{d}{d t} V_{i}(t)+D^{\prime}(t) S P_{i}(t)+D(t) \frac{d}{d t} S P_{i}(t)
$$

and using (2.2)-(2.3) and (4.6) we get after some simple arithmetic the equation

$$
\frac{d}{d t} V_{i}^{*}(t)=\delta^{0} V_{i}^{*}(t)+\pi_{i}(t)-b_{i}^{*}(t)-\sum_{j \neq i} \mu_{i j}^{0}(t)\left[V_{j}^{*}(t)+B_{i j}^{*}(t)-V_{i}^{*}(t)\right]
$$

\section{EXAMPLES}

To illustrate some of the results, we shall consider two examples: A singlepremium annuity policy and a disability policy. The first example focuses on ways of distributing interest surplus, whereas the other example is a discussion of surplus distribution in a three-state model. We have not included an example of a typical endowment policy, because we feel that the two other examples are more interesting.

\subsection{An annuity policy}

Let us consider a single-premium annuity policy where a benefit $b$ is paid continuously throughout the life of an individual $(x)$. The first order premium 
reserve is

$$
V(t)=b \bar{a}_{x+t}, \quad t \geq 0,
$$

using standard actuarial notation. We assume that the actual force of interest is a constant $\delta^{0}>\delta$ and that the interest earnings are the only source of surplus, i.e. $\mu^{0}(\cdot)=\mu(\cdot)$.

Then, according to (3.1), surplus is accumulated at the rate $\gamma(t)=\Delta \delta V(t)$, and we may, therefore, pay the insured the adjusted benefit

$$
b_{1}(t)=b+\Delta \delta V(t) .
$$

Alternatively, (4.8) shows that the surplus may also be distributed by means of the increased benefits

$$
b_{2}(t)=\exp \left[\left(\delta^{0}-\delta\right) t\right] b .
$$

It is interesting to note that (5.1) is typically a decreasing function of time/age, whereas (5.2) is increasing exponentially. Thus, the two formulas represent two completely different ways of distributing the same surplus.

In practice, however, it is not possible to adjust the benefits continuously as it is assumed in (5.1) and (5.2). In Denmark, for instance, pensions are adjusted only annually. Therefore, there is a need for more practical versions of (5.1) and (5.2). If, for example, the total surplus accumulated during year $t$, $t=0,1, \ldots$, has to be distributed through a level benefit $b_{3}(t)$ payable continuously during year $t$, then $b_{3}(t)$ has to be determined by

$$
V(t)=b_{3}(t) \bar{a}_{x+t: \overline{1}}^{0}+v^{0} p_{x+t} V(t+1), \quad t=0,1, \ldots,
$$

where the superscript " 0 " indicates that the values are based on $\delta^{0}$. Hence, $b_{3}(0)$ is the level benefit that is paid continuously during year $0, b_{3}(1)$ is paid during year 1 etc. It follows from (5.3) that the series of benefits $b_{3}(0)$, $b_{3}(1), \ldots$ serves the same purpose as the function $b_{1}(\cdot)$.

Similarly, the function $b_{2}(\cdot)$ may be replaced by level annual benefits in the following way. Assume that the benefit is a level amount $b_{4}(t)$ during year $t$. Then $b_{4}(t+1)$ is determined by the equation

$$
\begin{aligned}
& b_{4}(t) \int_{t}^{t+1} e^{-\delta^{0}(s-t)}{ }_{s-t} p_{x+t} \Delta \delta \bar{a}_{x+s} d s+v^{0} p_{x+t} b_{4}(t) \bar{a}_{x+t+1} \\
& =v^{0} p_{x+t} b_{4}(t+1) \bar{a}_{x+t+1} .
\end{aligned}
$$

Thus, we see that the surplus accumulated over the year is used to grant an increase of the benefit from $b_{4}(t)$ to $b_{4}(t+1)$.

Table 1 gives examples for an annuity of 10,000 issued to a male aged 60 . The valuation rate of interest is $4.5 \%, \delta=\log (1.045)$, whereas the actual interest rate is assumed to be $8 \%$, i.e. $\delta^{0}=\log (1.08)$. Moreover, the mortality is $\mu(t)=0.0005+10^{0.038(x+t)-4.12}$ which is the standard assumption used by Danish life companies. 
TABLE 1

COMPARISON OF VARIOUS WAYS OF DISTRIBUTING SURPLUS FOR AN ANNUITY OF 10,000 ISSUED TO A MALE AGED 60

\begin{tabular}{ccccc}
\hline \hline $\begin{array}{c}\text { Age } \\
x+t\end{array}$ & $b_{1}(t)$ & $b_{2}(t)$ & $b_{3}(t)$ & $b_{4}(t)$ \\
\hline 60 & 13,885 & 10,000 & 13,835 & 10,000 \\
61 & 13,784 & 10,335 & 13,734 & 10,350 \\
62 & 13,682 & 10,681 & 13,632 & 10,713 \\
63 & 13,580 & 11,039 & 13,529 & 11,089 \\
64 & 13,477 & 11,409 & 13,426 & 11,479 \\
65 & 13,373 & 11,791 & 13,322 & 11,884 \\
70 & 12,853 & 13,902 & 12,803 & 14,141 \\
75 & 12,345 & 16,391 & 12,297 & 16,861 \\
80 & 11,869 & 19,326 & 11,825 & 20,161 \\
\hline
\end{tabular}

The table highlights the difference between the payment schemes $b_{3}(t)$ and $b_{4}(t)$. The calculations show that $b_{3}(t)$ is larger than $b_{4}(t)$ during the first 8 years after which $b_{4}(t)$ exceeds $b_{3}(t)$. The distribution method that leads to $b_{4}(t)$ is widely used in Denmark, primarily because it provides some protection against inflation. However, one might also argue that in years with low inflation, many retirees are presumably prepared to forfeit inflation protection in return for higher benefits while they are healthy and the quality of life is higher. Thus, $b_{3}(t)$ should perhaps be recommended more widely than it has been until now.

\subsection{A disability policy}

We shall in this section consider an $n$-year disability policy issued on an able male aged $x$. The policy may be described by the three-state Markov model depicted in Figure 1. It is assumed that the policy provides a continuous

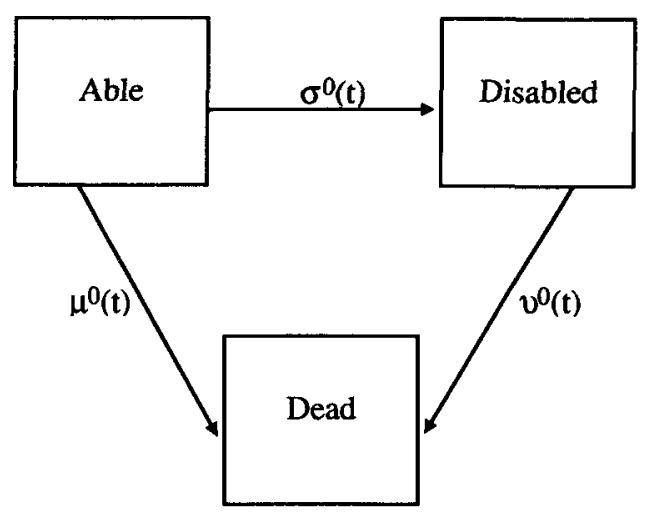

Figure 1 . The disability model. 
annuity of 1 as long as the insured is disabled. Premiums are waived during disability, and it is assumed that the premium payments cease after $m=n-5$ years in order to avoid negative reserves close to maturity.

Danish companies assume in their valuations that the transition intensities are given by

$$
\mu(t)=v(t)=0.0005+10^{0.038(x+t)-4.12}
$$

and

$$
\sigma(t)=0.0004+10^{0.060(x+t)-5.46} .
$$

The rate of interest is still assumed to be $4.5 \%$, i.e. $\delta=\log (1.045)$. We shall study surplus distribution under the somewhat more realistic assumptions that the actual behaviour of the policy is governed by

$$
\begin{aligned}
& \mu^{0}(t)=\theta_{1} \mu(t), \\
& \sigma^{0}(t)=\theta_{2} \sigma(t),
\end{aligned}
$$

and

$$
v^{0}(t)=\theta_{3} v(t),
$$

where $\underline{\theta}=\left(\theta_{1}, \theta_{2}, \theta_{3}\right)$ is given below. Moreover, the actual rate of interest is also in this example $8 \%$, i.e. $\delta^{0}=\log (1.08)$.

The premium $\pi$ and the first order reserves are given by

$$
\begin{gathered}
\pi=\bar{a}_{x: \bar{n}}^{a i} / \bar{a}_{x: \bar{m} \mid}^{a a}, \\
V_{a}(t)=\bar{a}_{x+t: \overline{n-t} \mid}^{a i}-\pi \bar{a}_{x+t: \overline{m-t} \mid}^{a a}, \\
V_{i}(t)=\bar{a}_{x+t: \overline{n-t},}^{i i},
\end{gathered}
$$

where $\bar{a}_{x: \bar{n} \mid}^{a i}=\int_{0}^{n} v^{s}{ }_{s} p_{x}^{a i} d s, \bar{a}_{x: \bar{n} \mid}^{a a}=\int_{0}^{n} v^{s}{ }_{s} p_{x}^{a a} d s, \bar{a}_{x: \bar{n} \mid}^{i i}=\bar{a}_{x: \bar{n} \mid}=\int_{0}^{n} v^{s}{ }_{s} p_{x} d s$,

and where ${ }_{s} p_{x}^{a a}=\exp \left(-\int_{0}^{s} \mu(u)+\sigma(u) d u\right),{ }_{s} p_{x}=\exp \left(-\int_{0}^{s} \mu(u) d u\right)$,

and ${ }_{s} p_{x}^{a i}={ }_{s} p_{x}-{ }_{s} p_{x}^{a a}$. The corresponding amounts at risk are $R_{a i}(t)=$ $V_{i}(t)-V_{a}(t), R_{a d}(t)=-V_{a}(t)$, and $R_{i d}(t)=-V_{i}(t)$. Here $a$ denotes the state able, $i$ the state disabled (invalid), and $d$ the state dead.

According to (3.1), surplus accumulates at the rates

$$
\begin{aligned}
\gamma_{a}(t) & =\Delta \delta V_{a}(t)+\Delta \sigma(t) R_{a i}(t)+\Delta \mu(t) R_{a d}(t) \\
& =(\Delta \delta-\Delta \mu(t)-\Delta \sigma(t)) V_{a}(t)+\Delta \sigma(t) V_{i}(t),
\end{aligned}
$$

and

$$
\begin{aligned}
\gamma_{i}(t) & =\Delta \delta V_{i}(t)+\Delta v(t) R_{i d}(t) \\
& =(\Delta \delta-\Delta v(t)) V_{i}(t)
\end{aligned}
$$


during stays in the states able and disabled, respectively. Here $\Delta \delta=\delta^{0}-\delta$, $\Delta \mu(t)=\mu(t)-\mu^{0}(t), \Delta \sigma(t)=\sigma(t)-\sigma^{0}(t)$, and. $\Delta v(t)=v(t)-v^{0}(t)$. Hence, the present values at time 0 of the total accumulated surpluses are

$$
\begin{aligned}
& \Gamma_{a}(n)=\int_{0}^{n} \exp \left(-\delta^{0} s\right){ }_{s} p_{x}^{0 a a} \gamma_{a}(s) d s, \\
& \Gamma_{i}(n)=\int_{0}^{n} \exp \left(-\delta^{0} s\right){ }_{s} p_{x}^{0 a i} \gamma_{i}(s) d s,
\end{aligned}
$$

and

$$
\Gamma(n)=\Gamma_{a}(n)+\Gamma_{i}(n),
$$

cf. (3.2). Here, ${ }_{s} p_{x}^{0 a a}$ and ${ }_{s} p_{x}^{0 a i}$ are second order values of ${ }_{s} p_{x}^{a a}$ and ${ }_{s} p_{x}^{a i}$, respectively. The corresponding possible terminal bonuses $T_{a}(n), T_{i}(n)$, and $T(n)$ are given by (4.2) and (4.3).

We have in Table 2 shown examples of (5.3)-(5.5) for policies with $x+n=65$ and $x+m=60$. Moreover, it is assumed in these examples that $\theta_{1}=0.7, \theta_{2}=0.8$, and $\theta_{3}=1$ which are close to what currently is used by many Danish companies. The figures illustrate clearly the size of the surplus inherent in the policies. Take as an example the policy issued at age 30. Here the actuarial present value of the total surplus is 0.144 compared with the total value of the premium payments $\pi \bar{a}_{x: \bar{m} \mid}^{a a}$ which equals 0.423 . The surplus might be distributed through the terminal dividends given in Table 2. However, it is hard to argue that only paying 2.13 and 5.12 to the lives that are able and disabled at age 65 is an equitable way of distributing the profit. It is also difficult to justify that large amounts should be paid to the disabled lives who have already collected benefits under the terms of the policy.

Table 3 shows for the example $x=30$ the possible benefits if the surplus is used to continuously increase the benefits. We have shown the rates of surplus accumulation $\gamma_{a}^{*}(t)$ and $\gamma_{i}^{*}(t)$, cf. (4.5), together with $1+D_{a}(t)$ and $1+D_{i}(t)$, respectively. Here $1+D_{a}(t)$ is the basic disability annuity that becomes payable if disability occurs at time $t$. This quantity and $\gamma_{a}^{*}(t)$ have been calculated assuming that the policy has remained in the state able during $[0, t)$. Similarly,

TABLE 2

EXAMPLES OF PRESENT VALUES OF ACCUMULATED SURPLUSES AND POSSIBLE TERMINAL BONUSES FOR VARIOUS DISABILITY POLICIES WITH $\underline{\theta}=(0.7,0.8,1)$

\begin{tabular}{cccccccc}
\hline \hline $\begin{array}{c}\text { Issue } \\
\text { age }\end{array}$ & $1000 \pi$ & $\Gamma_{a}(n)$ & $\Gamma_{i}(n)$ & $\Gamma(n)$ & $T_{a}(n)$ & $T_{i}(n)$ & $T(n)$ \\
\hline 20 & 19.0 & 0.086 & 0.037 & 0.123 & 3.97 & 9.29 & 5.65 \\
30 & 26.8 & 0.101 & 0.043 & 0.144 & 2.13 & 5.12 & 3.03 \\
40 & 40.8 & 0.110 & 0.049 & 0.159 & 1.05 & 2.77 & 1.51 \\
50 & 65.5 & 0.103 & 0.040 & 0.143 & 0.43 & 1.13 & 0.60 \\
\hline
\end{tabular}


TABLE 3

RATES OF SURPLUS ACCUMULATION AND SIZE OF INCREASED BENEFITS. AGE AT ISSUE $x=30$ AND $\underline{\theta}=(0.7,0.8,1)$

\begin{tabular}{lllcc}
\hline \hline $\begin{array}{l}\text { Age } \\
x+t\end{array}$ & $\gamma_{a}^{*}(t)$ & $\gamma_{i}^{*}(t)$ & $1+D_{a}(t)$ & $1+D_{i}(t)$ \\
\hline 30 & 0.002 & 0.560 & 1.00 & 1.00 \\
40 & 0.012 & 0.654 & 1.14 & 1.39 \\
50 & 0.029 & 0.655 & 1.51 & 1.93 \\
60 & 0.045 & 0.381 & 2.68 & 2.69 \\
61 & 0.044 & 0.324 & 2.97 & 2.78 \\
62 & 0.041 & 0.258 & 3.38 & 2.87 \\
63 & 0.036 & 0.183 & 4.02 & 2.97 \\
64 & 0.027 & 0.098 & 5.38 & 3.07 \\
64.5 & 0.019 & 0.050 & 7.17 & 3.12 \\
65 & 0 & 0 & $\infty$ & \\
\hline
\end{tabular}

$1+D_{i}(t)$ is the annuity payable at time $t$ and $\gamma_{i}^{*}(t)$ measures the rate of surplus accumulation, provided that the insured became disabled just after time 0 . It is interesting to note that (4.7) leads to

$$
D_{i}(t)=\int_{0}^{t} q_{i}(s) \exp \left(\int_{s}^{t} r_{i}(u) d u\right) d s=\exp \left(\int_{0}^{t} r_{i}(u) d u\right)-1
$$

with $q_{i}(s)=\gamma_{i}(s) / S P_{i}(s)=\Delta \delta-\Delta v(t), S P_{i}(t)=V_{i}(t)$, and $r_{i}(u)=q_{i}(u)$. Hence, $D_{i}(t)$ is in general easy to compute, and in the example in Table 3 $\Delta v(t)=0$, so $1+D_{i}(t)=\exp (\Delta \delta t)$, cf. (5.2).

It is interesting to note that $1+D_{a}(t)$ and $1+D_{i}(t)$ increase at different rates. In particular, the sharp increase in $1+D_{a}(t)$ close to maturity should be noted. Actually, it is easily seen that $1+D_{a}(t) \rightarrow \infty$ as $t \rightarrow n$. It may be explained by the fact that close to maturity, the surplus is of the size $O(h)$, $h=n-t$, whereas the price of providing additional benefits is $\bar{a}_{x+t: \overline{n-t} \mid}^{a i}=O\left(h^{2}\right)$. In practice, these excessive benefits should, of course, be avoided, and it may be achieved by shifting to a system with cash or deferred bonuses when the policy approaches maturity.

In Table $3,1+D_{i}(t)$ yields the annuity at time $t$ if the disability occurred at time 0 . However, if disability occurs at some later time, say $t_{i}$, then it follows from (4.7) that the benefit at time $t \geq t_{i}$ is given by

$$
\begin{aligned}
1+\tilde{D}_{i}(t) & =\left(1+D_{a}\left(t_{i}\right)\right) \exp \left(\int_{t_{i}}^{t} r_{i}(u) d u\right) \\
& =\left(1+D_{a}\left(t_{i}\right)\right)\left(1+D_{i}(t)\right) /\left(1+D_{i}\left(t_{i}\right)\right) .
\end{aligned}
$$

Thus, if for example disability occurs at age 40 , then the initial annuity is 1.14 , which after 10 years of disability will have risen to $(1.14)(1.93) / 1.39=1.58$. It illustrates that the benefits while disabled depend on the duration of the disability. 
TABLE 4

EXAMPLeS OF Disability ANNUITIES $1+D_{i}(t)$ IN THE SITUATIONS Where $\theta_{3}=1,2$ AND 5 . AGE AT ISSUE $x=30$ AND $\left(\theta_{1}, \theta_{2}\right)=(0.7,0.8)$

\begin{tabular}{cccc}
\hline \hline $\begin{array}{c}\text { Age } \\
x+t\end{array}$ & $\theta_{3}=1$ & $\theta_{3}=2$ & $\theta_{3}=5$ \\
\hline 30 & 1.00 & 1.00 & 1.00 \\
40 & 1.39 & 1.42 & 1.52 \\
50 & 1.93 & 2.07 & 2.53 \\
60 & 2.69 & 3.18 & 5.27 \\
65 & 3.17 & 4.11 & 9.01 \\
\hline
\end{tabular}

TABLE 5

Present Values of aCcumulated SURPLUSes for Different Values of $\underline{\theta}$. AGE AT ISSUE $x=30$

\begin{tabular}{cccc}
\hline \hline$\underline{\theta}=\left(\theta_{1}, \theta_{2}, \theta_{3}\right)$ & $\Gamma_{a}(n)$ & $\Gamma_{i}(n)$ & $\Gamma(n)$ \\
\hline$(0.7,0.8,1)$ & 0.101 & 0.043 & 0.144 \\
$(0.7,1,1)$ & 0.051 & 0.054 & 0.104 \\
$(0.7,1,2)$ & 0.051 & 0.062 & 0.113 \\
$(0.7,1,5)$ & 0.051 & 0.085 & 0.136 \\
$(0.7,1,10)$ & 0.051 & 0.112 & 0.163 \\
\hline
\end{tabular}

We have also shown in Table 4 the kind of disability annuities that can be offered if it is further taken into account that disabled lives often have a much higher mortality than able lives. We have shown examples of $1+D_{i}(t)$ in the situations where $\theta_{3}=1,2$, and 5 . Otherwise, the assumptions are the same as in Table 3. It is clear that substantial mortality gains on the disabled lives might be used to increase the disability benefits further.

However, in some cases mortality gains on disabled lives would rather be used to offset unsatisfactory disability experience among able lives. In this way all get a share of the "favourable" mortality among disabled lives. To give an impression of to what extent an unfavourable value of $\theta_{2}$ can be offset by a favourable value of $\theta_{3}$, we have shown in Table 5 some examples where $\theta_{2}=0.8$ and 1, and where $\theta_{3}=1,2,5$, and 10 . Hence, taking $\underline{\theta}=\left(\theta_{1}, \theta_{2}, \theta_{3}\right)=(0.7,0.8,1)$ as our basis, it is seen that even $\theta_{3}=5$ is not sufficient to eliminate the overall effect of $\theta_{2}=1$, whereas $\theta_{3}=10$ more than compensates for the effect of $\theta_{2}=1$.

\section{REFERENCES}

Berger, A. (1939) Mathematik der Lebensversicherung. Springer, Wien.

HoEM, J.M. (1969) Markov chain models in life insurance. Blätter der Deutschen Gesellschaft für Versicherungsmathematik IX, 97-107.

HOEM, J.M. (1988) The versatility of the Markov chain as a tool in the mathematics of life insurance. Transactions of the 23rd international congress of actuaries, vol. R, 171-202. 
Ramlau-Hansen, H. (1988) The emergence of profit in life insurance. Insurance: Mathematics and Economics 7, 225-236.

Simonsen, W. (1970) Forsikringsmatematik, hefte III. Københavns Universitets Fond til tilvejebringelse af laremidler.

SVERDRUP, E. (1969) Noen forsikringsmatematiske emner. Statistical memoirs No. 1, Institute of Mathematics, University of Oslo.

HENRIK RAMLAU-HANSEN

Baltica Insurance Company Ltd., Klausdalsbrovej 601, DK-2750 Ballerup, Denmark. 